\title{
Utilization of Encoding Techniques at the Signal Transmission in the Optical Fiber
}

\author{
Filip Čertík
}

Institute of Telecommunications, FEI, Slovak University of Technology in Bratislava, Slovakia

Copyright (C)2015 Horizon Research Publishing All rights reserved.

\begin{abstract}
This paper presents a possible utilization of advances signal processing techniques in the optical transmission system at the signal transmission. A contribution briefly introduces basic characteristics of the optical fiber, Forward Error Correction techniques and digital modulation formats considered for analysis. The paper mainly focuses on RS and $\mathrm{BCH}$ codes used in conjunction with OOK and BPSK techniques and their practical exploitation in the optical transmission system. Then, a created simulation program for the optical transmission system is presented with detailed description of blocks related to mutual cooperation RS and $\mathrm{BCH}$ codes with binary modulation techniques (OOK, DBPSK and BPSK). In the third part, more detailed analyses of $\mathrm{RS}$ and $\mathrm{BCH}$ performances are presented.
\end{abstract}

Keywords Optical Transmission Path, RS Code, BCH Code, Optical Simulation Program, Binary Modulation Techniques

\section{Introduction}

Nowadays, an interest in the signal transmission through optical fibers are rapidly increasing due to the transmission bandwidth. Constructing new optical transmission paths can be time consuming, expensive and sometimes not available solution. In the electric domain, utilizing new high-speed modulators or using error-correcting codes can lead to increasing of the transmission capacity. Such solutions can be easily integrated. With increasing of modulation rates, linear and nonlinear influences on transmitted optical signal are growing and by this way additional bit errors in information signals are generating. Therefore, it is important to choose error correction codes that ensure a desirable value of the Bit Error Rate (BER). This paper presents a possible utilization of combination of encoding and modulation in the optical transmission system. For analysis, simulations of the signal transmission in the optical fiber using MATLAB Simulation tools are utilized [1,2]. First, basic characteristics of the optical fiber are introduced. When the DWDM technology is used to provide the highest possible capacity of the optical system, then nonlinear effects present in the optical transmission medium become more relevant. In the next part, the simulation program for presenting optical signals passing through the optical transmission path influenced by linear and nonlinear effects is described. In the final part, the RS and $\mathrm{BCH}$ codes are used with different binary modulation techniques to improve the BER performance. The comparison of different error codes with modulation techniques is presented.

\section{Characterizations of the optical transmission system}

\subsection{The Optical Path Parameters}

For executed analysis, a basics of the optical effects are presented by the optical transmission medium. Each optical fiber represents a transmission system, which is frequency dependent. A pulse propagation inside this transmission system can be described by the nonlinear Schrödinger equation (NLSE), which is derivate from Maxwell equations. From the NLSE equation we can expresses effects in optical fibers that can be classified as $[3,4]$ :

- linear effects, which are wavelength depended,

- nonlinear effects, which are intensity (power) depended.

Major impairments of optical signals transmitted via optical fiber are mainly caused by linear effects - the dispersion and the attenuation. The attenuation limits power of optical signals and represents transmission losses. Nowadays, optical transmission systems are able to minimize impact of the attenuation by deploying regenerators or all optical amplifiers like Raman or EDFA amplifiers. Another source of linear effects represents the dispersion that causes broadening of optical pulses in time and phase shifting of signals at the fiber end. There are three dispersion types [3-7]:

- modal dispersion caused by the different propagation velocity of signal modes in multimode fibers.

- chromatic dispersion caused by the different propagation velocity of signal wavelengths from a laser source via optical fibers.

- polarization mode dispersion caused by the birefringence effect of nonsymmetrical and imperfect optical fibers. 
The modal dispersion occurs only in multi mode fibers that are mainly used for short distances. Therefore it will not be discuss in this paper. The $\mathrm{CD}$ is caused by the fact that it is impossible to create monochromatic light source. Each pulse wavelength transmits with different velocity that leads to broadening and phase shifting at the fiber end. The PMD is a random phenomenon that can be only statistically evaluated. The complexity of this random phenomenon often leads to incorrect measurements and simulations of the PMD effect. The light mode transferred via the single mode optical fiber consists of two modes on different polarization planes and the PMD is caused by different velocity of these two polarization modes. In the simulation model, the $\mathrm{CD}$ and the PMD are realized in following block shown on fig. 1 and fig. 2. The Group Velocity Delay $G V D$ and differential group delay $(D G D)$ is used to characterize and simulate the $\mathrm{CD}$ and PMD effects. More detailed analysis of linear effects is published in [5].

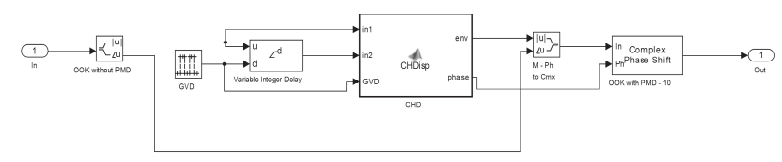

Figure 1. Chromatic Dispersion block

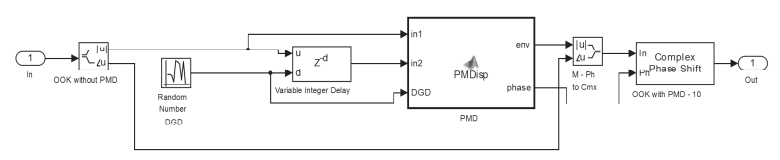

Figure 2. Polarization Mode Dispersion block

The nonlinear effects play an important role in the optical signal transmission. We can classify nonlinear effects by following:

Kerr nonlinearities are self-induced effects, where the phase velocity of the pulse depending on the pulse's own intensity. The Kerr effect describes a change in the fiber refractive index due to electrical perturbations [7]. Due to the Kerr effect, we are able to describe following effects:

- Self-phase Modulation (SPM) - effect that changes the refractive index of the transmission medium caused by intensity of the pulse.

- Four Wave Mixing (FWM) - effect where a fourth wave can be arised by mixing of optical signals and can appear in the same wavelength as one of the mixed wave.

- Cross-phase effect (XPM) - effect where an optical pulse can change the phase of another pulse with the different wavelength. This effect causes a spectral broadening.

Scattering nonlinearities occur due to a photon inelastic scattering to lower energy photons. The pulse energy is transferred to another wave with a different wavelength. Two effects appear in the optical fiber:

- Stimulated Brillouin Scattering (SBS) and Stimulated Raman Scattering (SRS) - effects that change variance of light wave into different waves when the intensity reaches certain threshold [5-9]
In the simulation model, the Kerr nonlinearities (FWM, SPM and XPM) realized in following blocks are shown on fig. 3 and fig. 4 and the scattering nonlinearities (SBS and SRS) are shown on fig. 5. The FWM only occurs in WDM systems and it mainly depends on power of adjacent-channel neighbors, wavelength channel spacing and dispersion. The SPM effect change signal phase and frequency depending on signal intensity changes. The XPM effect is similar to SPM and depends on signal intensity changes of adjacent-channel neighbors. Affection of the signal intensity caused by SBS and SRS change the envelope of signal and impact the Optical Signal to Noise Ratio OSNR. More detailed analysis of linear effects is published in [6,7].

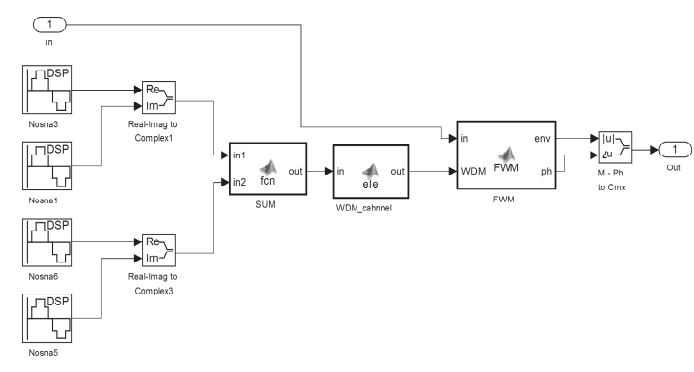

Figure 3. Four-Wave Mixing block

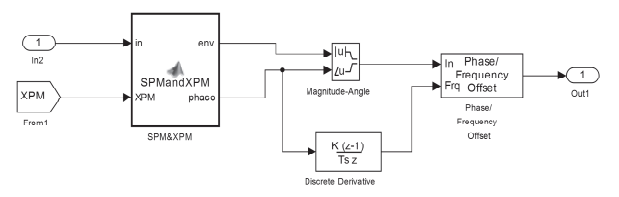

Figure 4. Self-Phase Modulation and Cross-Phase Modulation block

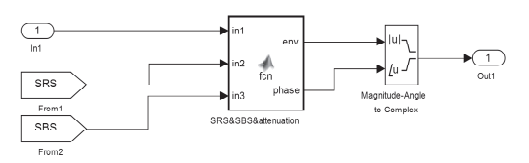

Figure 5. Stimulated Brilouin and Stimulated Raman Scattering block

\subsection{Forward Error Correction Techniques}

A forward error correction (FEC) techniques (also known as Error Correcting Code ECC) is a system, where additional data are inserted to data message so that it can be recovered by a receiver even when a number of errors due to transmission occurs. These FEC codes are widely used in systems where retransmission of the data is not an option such as broadcasting and high haul optical transmission systems. FEC codes are usually distinguished between convolutional and block codes:

- Convolutional codes that are processed on a bit-by-bit basis.

- Block codes that are processed on a block-by-block basis.

For analysis, block codes, especially cyclic block codes are considered. Cyclic block codes are widely used in data communication because their structure makes encoder and decoder circuitry simple. Cyclic block codes are defined as the 
cyclic $(n, k)$ code if $C$ is a linear code of length $n$ over a finite field and if any cyclic shift of a codeword is also a codeword [10-12] as shown in:

$$
\left(c_{0}, c_{1}, \ldots, c_{n-1}\right) \in C \Leftrightarrow\left(c_{n-1}, c_{0}, c_{1}, \ldots, c_{n-2}\right) \in C
$$

The information data with length $k$ are coded with polynomial $g(x)$ using (2).

$$
c(x)=i(x) g(x)
$$

where $c(x)$ represents polynomial with degree $(n-1), i(x)$ is the information polynomial of degree $(k-1)$ and the generator polynomial $g(x)$ must be of degree $(n-k)$. The ReedSolomon codes that belong to cyclic block codes are widely used in many communication fields. The RS codes are related with $\mathrm{BCH}$ codes and can be defined as a primitive $\mathrm{BCH}$ code of length $n$, where:

$$
n=q-1=2^{s}-1 \quad \text { over } \quad G F(q)=G F\left(2^{s}\right)
$$

RS codes are specified as $R S(n, k)$ or $R S(n, k, d)$, where $n$ represents code length, $k$ represents information length and $d$ is hamming distance. Let $t$ is number of errors that can be corrected, then (4) shows the correction $(t)$ or detection (2.t) ability for the specified code [10-13]. The minimum Hamming distance $d_{m i n}$ shows the maximum possibility of the code error correction ability defined by (5).

$$
\begin{gathered}
2 t=(n-k) \\
t=\frac{n-k}{2} \geq d_{\text {min }}
\end{gathered}
$$

Assuming RS code with length $n=q-1$ over $\mathrm{GF}(q)$ finite field with $q$ elements and assuming $\alpha$ to be a primitive $n$ th root of unity of $\operatorname{GF}(q)$ where $d$ falls into the interval 1 $\leq d \leq n$ then the generator polynomial $g(x)$ is given by:

$$
g(x)=\prod_{i=1}^{d-1}\left(x-\alpha^{i}\right)=(x-\alpha)\left(x-\alpha^{2}\right) \ldots\left(x-\alpha^{d-1}\right)
$$

There exist two coding types for RS codes:

- nonsystematic: $c(x)=i(x) g(x)$

- systematic: $c(x)=i(x) x^{n-k}+i(x) x^{n-k} \bmod g(x)$

The decoding for RS codes is based on syndrome equations:

$$
s_{k}=\sum_{i=1}^{t} Y_{i} X_{i}^{k} ; \quad k=0,1, \ldots, 2 t-1
$$

where $X_{i}$ is locator $i$-th error and $Y_{i}$ represents its value (detection of location and value). More information about RS encoding techniques can be found in [11-13].

The Bose Chaudhuri Hocquenghem $\mathrm{BCH}$ code is a cyclic polynomial code over a finite field with chosen polynomial generator. $\mathrm{BCH}$ codes are $t$-error correcting codes defined over finite fields $\operatorname{GF}(q)$, where $2 t+1<q$. The advantage of $\mathrm{BCH}$ codes is using syndrome to decode errors in which there exist good decoding algorithms that correct multiple errors [10-13]. The generating of a binary $\mathrm{BCH}$ code over an extension field $\mathrm{GF}\left(q^{m}\right)$ is easy to construct. The polynomial generator $g(x)$ is needed to obtain a cyclic code. For any integer $m \geq 3$ and $t<2^{m}-1$, there exists a primitive $\mathrm{BCH}$ code with parameters: $n=2^{m}-1, n-k \leq m . t, d_{\min } \leq 2 t+1$. The generator polynomial $g(x)$ of $t$-error-correcting primitive $\mathrm{BCH}$ codes of length $2^{m}-1$ is given by:

$$
g(x)=L C M\left\{m_{1}(x), m_{2}(x), \ldots, m_{2 t-1}(x), m_{2 t}(x)\right\}
$$

where LCM represent Least Common Multiple. Then the code is generated using (2). BCH codes are decoded with various algorithm based on calculation of syndromes values for the received codeword.

\subsection{Digital Modulation Formats}

The digital modulation represents the signal transformation in a way it adapts to the transmission medium. We can change the information signal by adding a carrier signal and therefore we can get new modulated signal that is suitable for a transmission via the optical transmission medium. There are four basic physical attributes to adapt to the optically transmitted data (carrier modulations) - intensity, phase, frequency and polarization. For these modulation techniques a sequence of digital symbols is used that influence determination parameters of a high frequency harmonic (sinusoidal) signal. An electric signal modulates the corresponding carrier optical signal (usually at the wavelength $1550 \mathrm{~nm}$ ) [12]. According to the parameter of the optical signal that is changed, we can divide digital modulations into these groups:

- Amplitude Shift Keying (ASK)

- Phase Shift Keying (PSK)

- Polarization Shift Keying (PolSK)

\section{- DUOBINARY}

In the simulation model, the OOK, the BPSK and the DBPSK are realized in following blocks of Data signal and MachZehnder Modulator MZM. This simulation uses MZM physical principles that are based on the interaction of two polarized waves (an interference of waves or a destruction of waves) and MZM block is shown on fig. 6. In a case of the OOK modulation, two linearly polarized waves with same polarization interfere. In a case of BPSK and DBPSK modulations, two waves are linearly polarized but there is the $\pi$ radian phase shift. In the first case, when an electric signal is present, then optical waves interfere each other creating wave propagating in the $\mathrm{z}$ direction. The absence of electric data signal, optical waves interfere to destructive state resulting to no signal. In a case of the PSK modulation, when an electric data signal is absent, optical waves interfere creating a polarized wave that propagates in the $\mathrm{z}$ direction with power $P$ and if the electric signal is present, waves interfere creating the same wave but the opposite polarization direction. The closer analysis of modulation techniques is explained in articles [12-15].

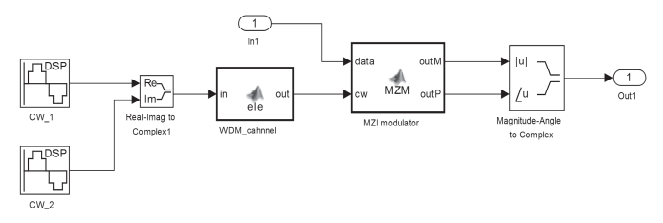

Figure 6. Mach-Zehnder Modulator block 


\section{The Simulation environment for the signal transmission in the optical fiber}

The presented simulation model comes out from the simulation model for optical communications introduced in [14]. A modeling is performed in Matlab Simulink 2014. The simulation model presents an influence of linear and nonlinear effects in the optical transmission media such as attenuation, chromatic and polarization mode dispersions, FWM, SPM and XPM and binary modulation and coding techniques. For executed analysis, the simulation model is realized for the Samsung single mode UltraPassTM Non-zero dispersionShifted optical fiber [15] that meets the specification ITU-T G.656 [16]. The optical transmission media simulation will be demonstrated on 3 modulation types - On/Off shift Keying (OOK), Binary Phase Shift Keying (BPSK), Differential Binary Phase Shift Keying (DBPSK) using RS and BCH codes [17]. Assuming the OOK modulation is noncoherent and uses direct detection. However, the phase modulations are coherent systems that are using local osciloscop to detect phases. The phase demodulation is shown on fig. 7 .

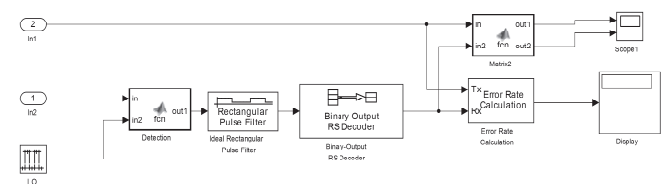

Figure 7. Phase demodulation block

The simulation scheme for 80 channels optical transmission WDM system with $320 \mathrm{~km}$ length and EDFA is shown on fig. 8 , which is the same analysis example with the 10 Gbit/s data rate. Each block represents individual subsystem as be described below. The Data signal block is used as a source of electric data signal and it also include encoder for specific cyclic code. The data signal scheme is similar to OOK and BPSK modulations and differs from the DBPSK modulation as it uses XOR function of actual bit and delayed bit. The Data block scheme signal is shown on fig. 9 and the $10 \mathrm{Gbit} / \mathrm{s}$ electric data signal is shown on fig. 10. As can be seen, the ideal signal is filtered to the Gaussian pulse. For describing and comparing various combination of formats, a bit error rate (BER) calculation and estimation from the EYE diagram are presented. The BER calculation is done by comparing input and output bits for each example. Because the BER calculation is a very time consuming process, we calculate the BER estimation of each approach. The EYE diagram displays each bit in the interval. In other words, the EYE diagram is an oscilloscope that displays a received signal repetitively sampled with the bit interval. The $10 \mathrm{~km}$ fiber length simulation does not include Erbium Doped Fiber Amplifiers EDFA as signal can be detected in detector. With a fiber length (more than $80 \mathrm{~km}$ ), the attenuation highly increasing limits the optical signal power to point where a detector is unable to detect it. Therefore, we can increase the optical signal intensity using the EDFA amplifier with the $12.8 \mathrm{~dB}$ gain and the $4 \mathrm{~dB}$ noise figure $N F[18,19]$. The gain represents an increased level of the optical power and the $N F$ represents a ratio of the following $S N R_{\text {in }}$ before amplification divided by $S N R_{\text {out }}$ after amplification. The BER estimation principle for the OOK modulation is shown on fig. 11 and for the
BPSK modulation on fig. 12. More detailed principles of the BER estimation from Q factor are shown in [20].

The first part of the analysis is focused on the BER values at the signal transmission in optical transmission medium under negative influences of linear and nonlinear effects. There are only OOK, BPSK and DBPSK modulations considered without any encoding techniques using $10 \mathrm{Gbit} / \mathrm{s}$ transmission rate. Operational optical transmission systems has to ensure that the BER value is less than $10^{-12}$ or $10^{-15}$ for successful transmission. The BER analysis of OOK is kept in boundaries for transmission less than $50 \mathrm{~km}$ as shown in fig. 13 .

The encoding techniques are used to improve the range of the optical transmission system. The RS code shows better performance than $\mathrm{BCH}$ code, both codes provide improvement of optical transmission system range to $80-90 \mathrm{~km}$. Modulations were coded with systematic RS code and both, $\mathrm{RS}$ and $\mathrm{BCH}$ codes were decoded with syndrome equation followed by (7). The $\mathrm{BCH}$ encoder is based on (8). Both phase modulations shows better performance that OOK modulation as shown in fig. 13. The CD and PMD mainly limit the OOK modulation. In case of BPSK and DBPSK modulation, the coherent system are capable to suppress dispersion increasing the optical transmission system range to around $100 \mathrm{~km}$ length. The best performance of optical system was acquired for BPSK modulation with RS code that increase the system to 160 - $170 \mathrm{~km}$. Similar results shows the DBPSK, where the optical system range varies $140-150 \mathrm{~km}$. The coding enhancement for digital binary modulations is shown in fig. 14.

\section{Conclusions}

The paper present analysis of advanced signal processing techniques in optical transmission systems. The simulation model of the optical transmission system was introduced that includes linear a nonlinear effects. The simulation results show the limitation of binary modulation techniques and their improvement using FEC codes. Both RS and $\mathrm{BCH}$ codes were used on OOK, BPSK and DBPSK modulation. The $\mathrm{RS}$ code performed better in all modulation schemes. The graph plotted between different modulation techniques with $\mathrm{RS}$ code and $\mathrm{BCH}$ code shows the performance gain of RS code over $\mathrm{BCH}$ code in optical transmission system. Acquired results from the initial analysis confirm the low performance of binary systems and that there is a sense for focusing on advanced optical signal processing techniques that highly improve the optical transmission system range. Comparing with the original OOK modulation using a combination of modulation formats (BPSK, DBPSK) and encoding techniques $(\mathrm{RS}, \mathrm{BCH})$ can bring increasing of optical transmission path. Also, an evaluation of BER parameters in existing optical transmission system can be realized after implementation of appropriate advanced signal processing techniques. In future analysis, we can design a new combination of high-bit rate modulation formats, such as QPSK, 8PSK, 16QAM and FSK with coding techniques and focus on new a encoding, such as LDPC codes. The suitable combination of modulation and new encoding techniques can enhance the performance of new optical systems and also improve range of existing system that are already deployed. 


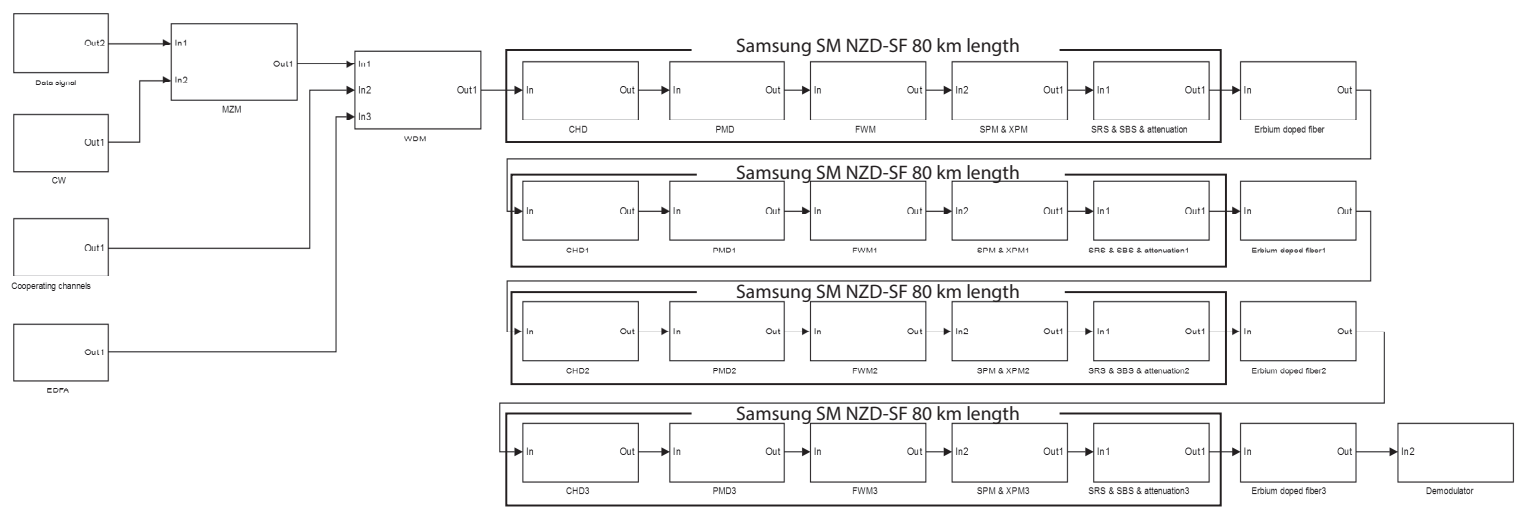

Figure 8. The simulation scheme for 80 channels optical transmission system with $320 \mathrm{~km}$ length using EDFA amplifiers

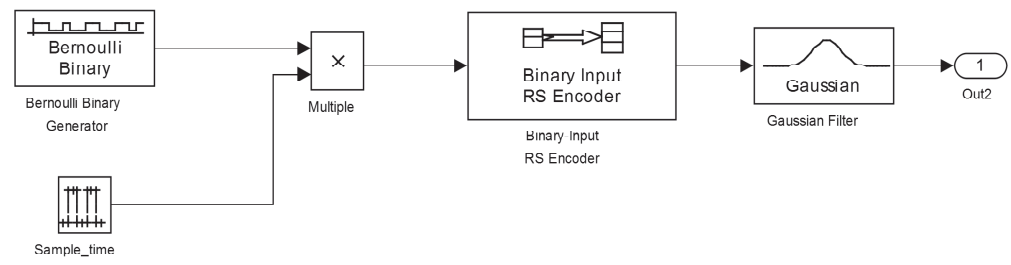

Figure 9. Data signal block scheme

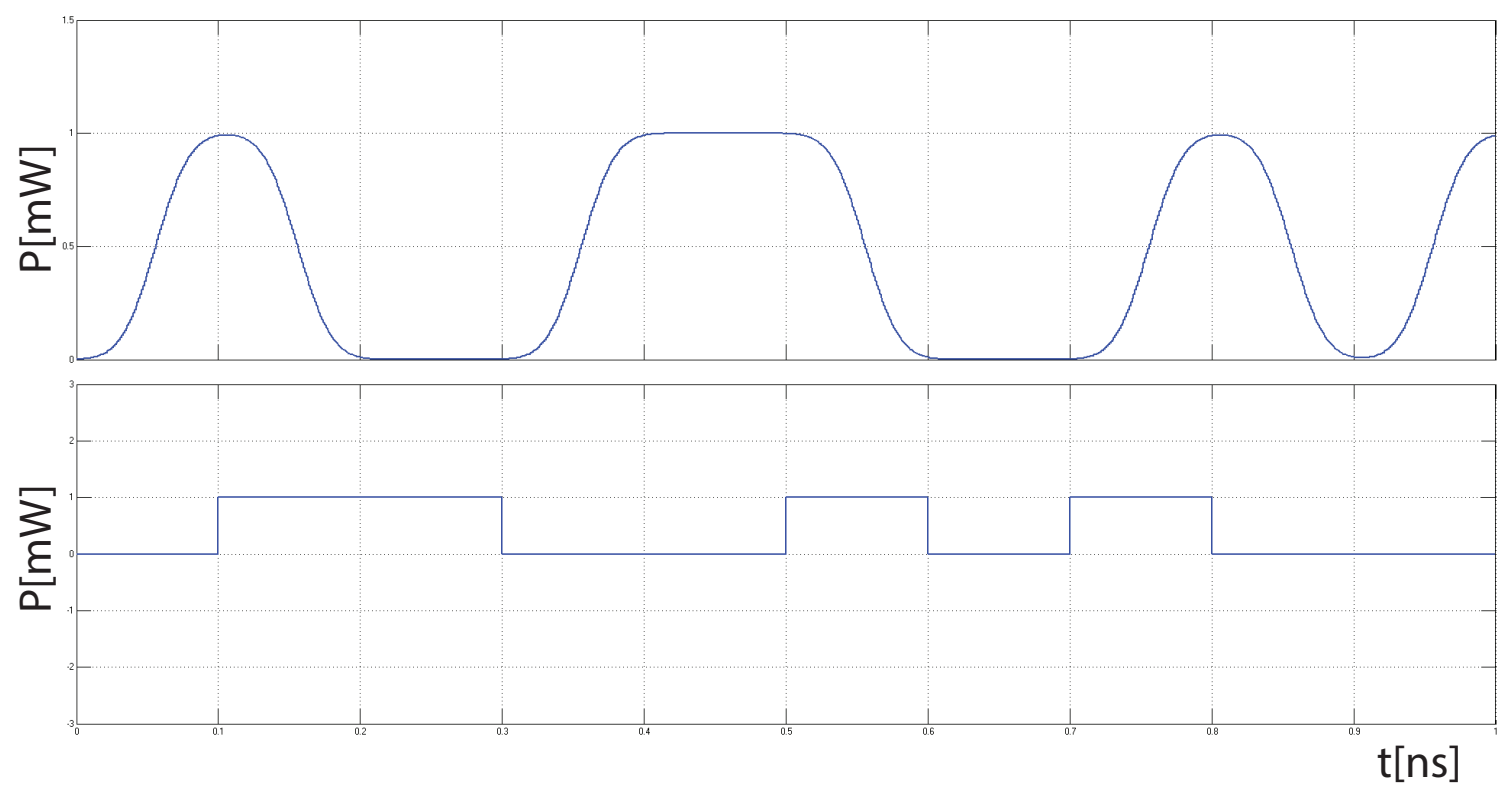

Figure 10. The generated Gaussian electric signal 


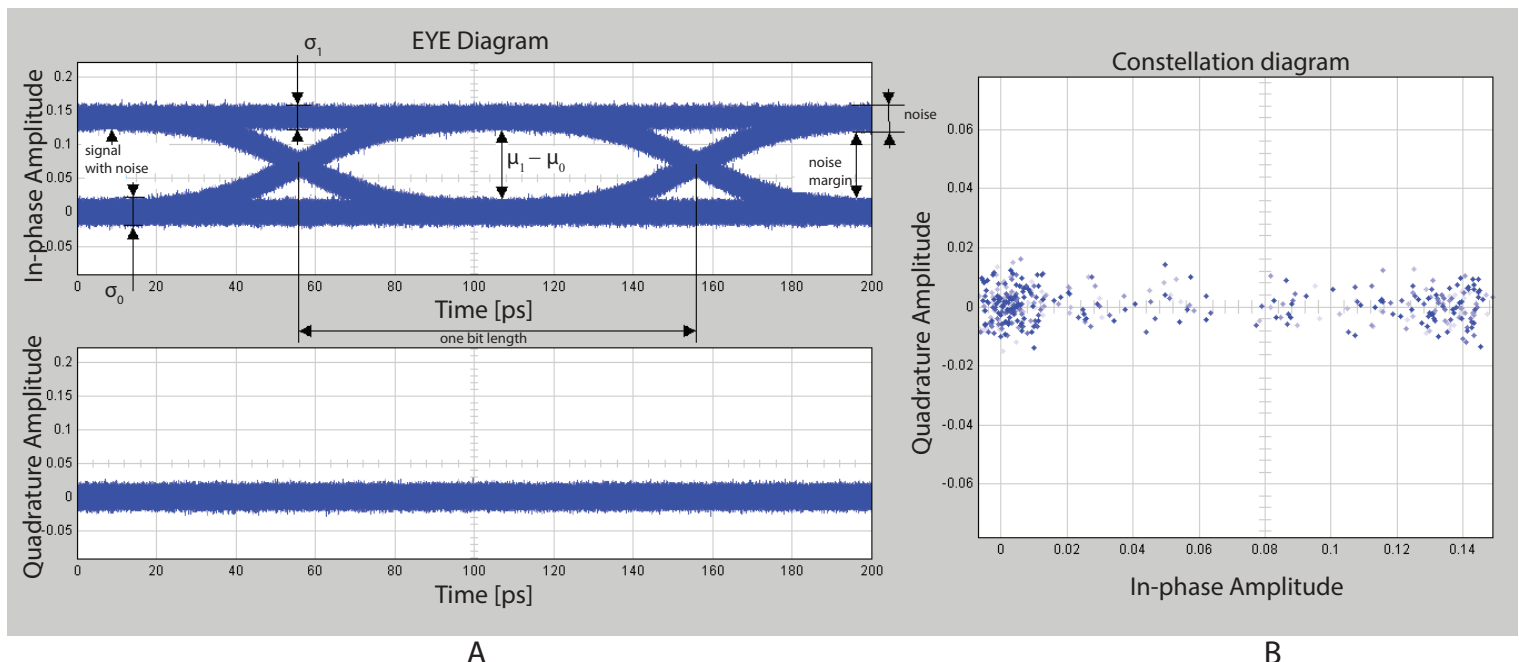

Figure 11. The EYE diagram (A) and the constellation diagram (B) for the OOK modulation

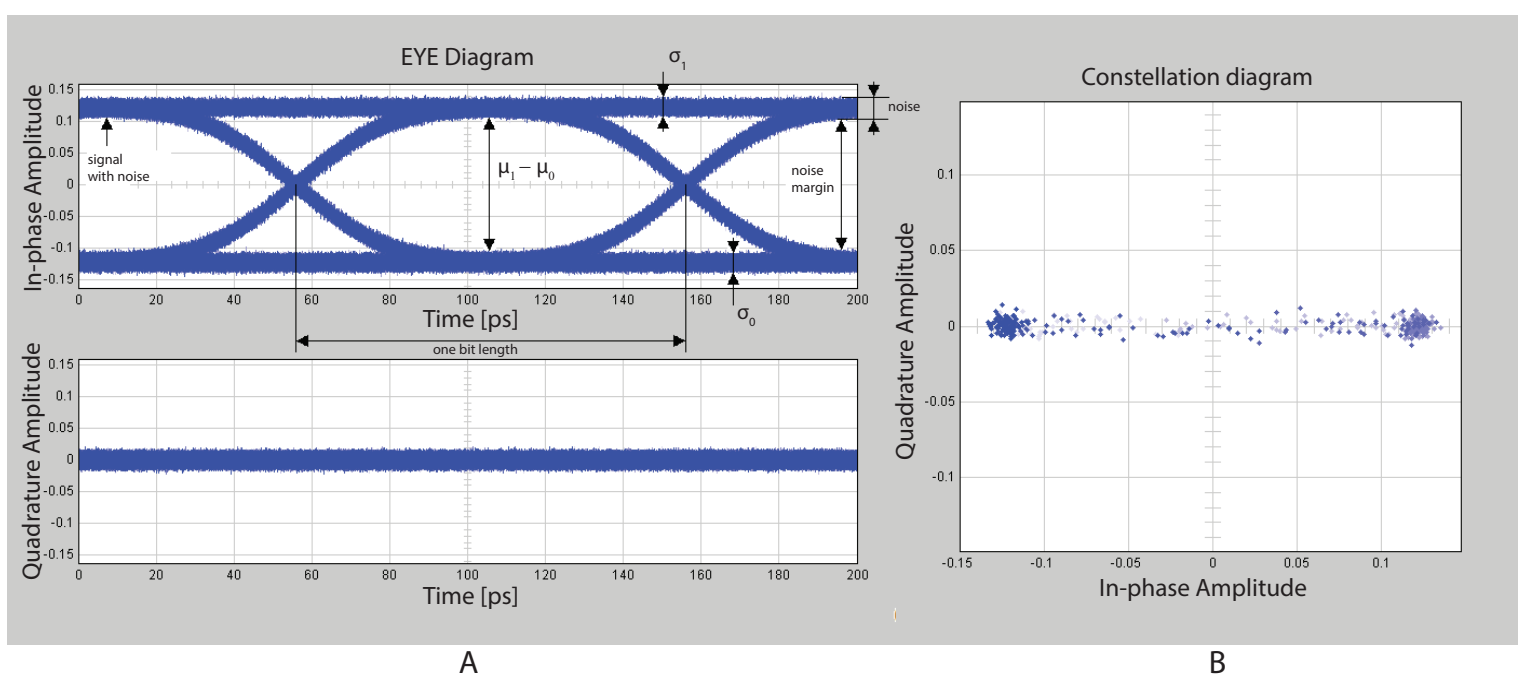

Figure 12. The EYE diagram (A) and the constellation diagram (B) for the BPSK modulation

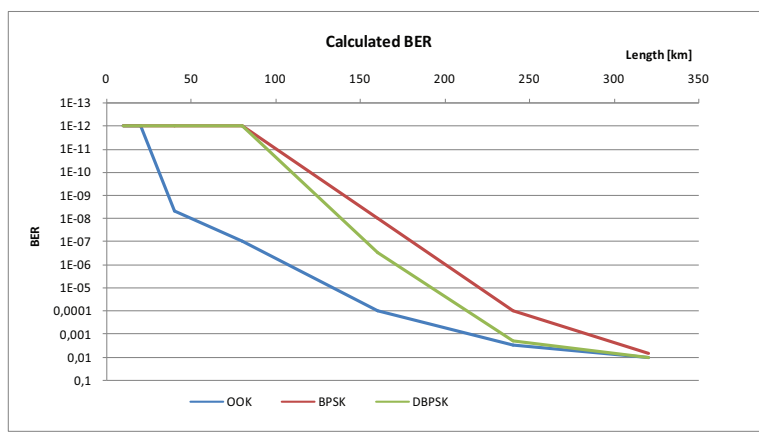

A

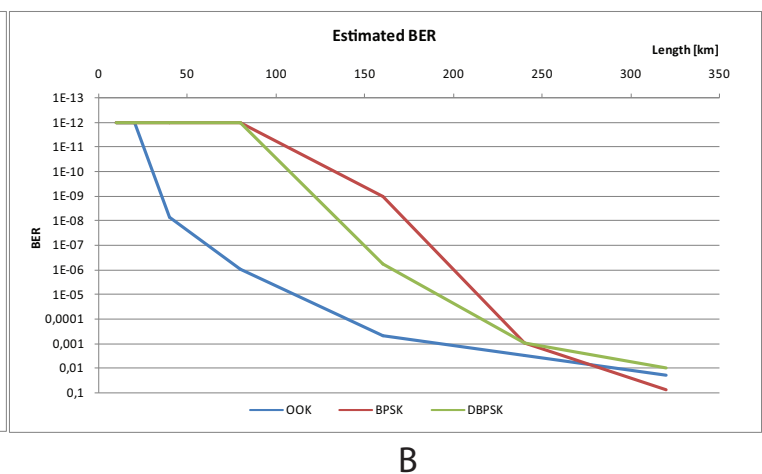

B

Figure 13. Calculated (A) and estimated(B) BER values 


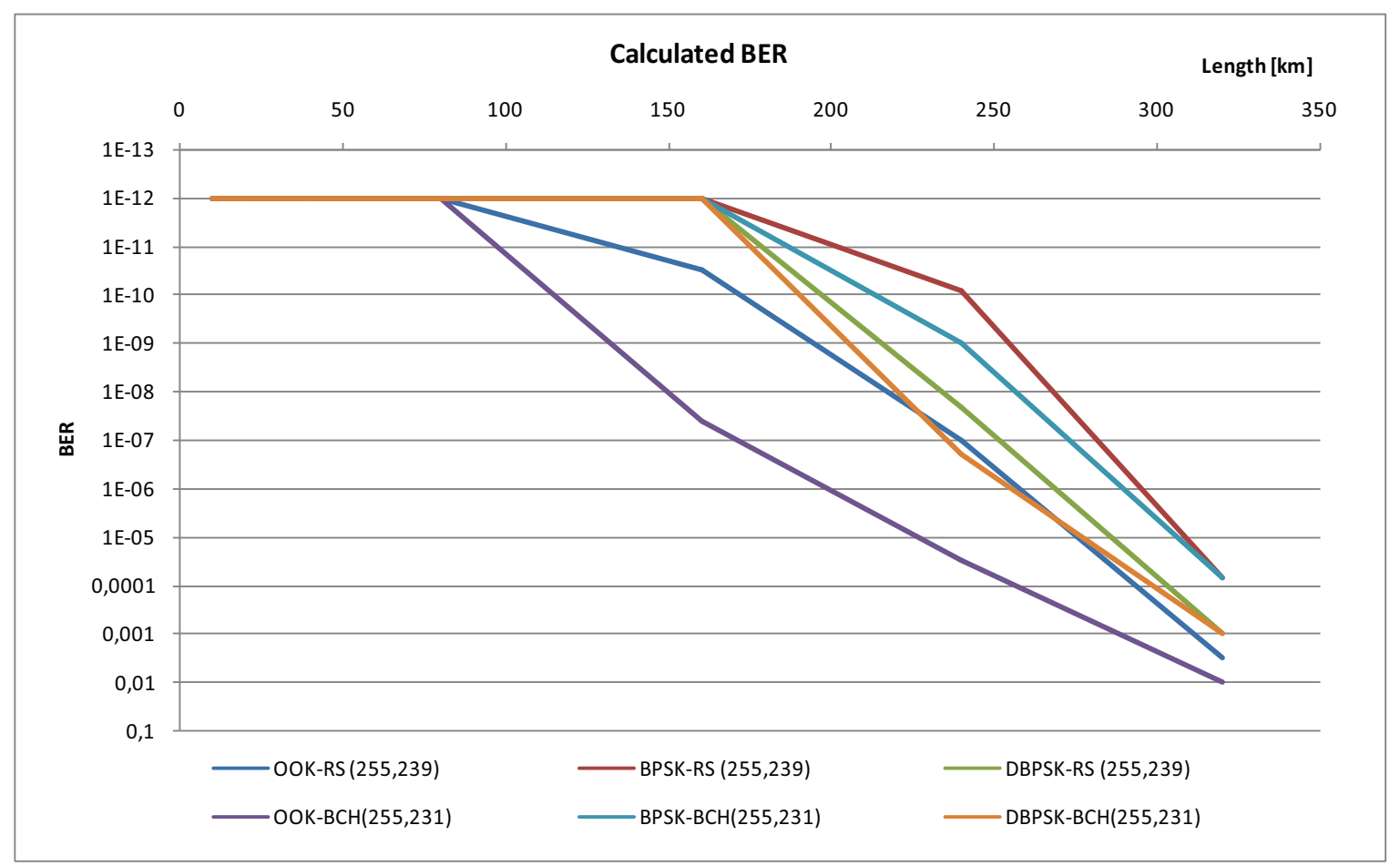

Figure 14. Bit error rate for different modulation with RS and BCH codes

\section{Acknowledgements}

This work is a part of research activities conducted at Slovak University of Technology Bratislava, Faculty of Electrical Engineering and Information Technology, Institute of Telecommunications, within the scope of the projects KEGA No. 039STU-4/2013 "Utilization of Web-based Training and Learning Systems at the Development of New Educational Programs in the Area of Optical Transmission Media”.

\section{REFERENCES}

[1] D. Petras, I. Baronak, and E. Chromy. Presence service in IMS, The Scientific World Journal, vol. 2013 (2013), Article ID 606790, 8 pages, ISSN: 1537-744X, 2013.

[2] E. Chromy, M.Jadron, and T. Behul. Admission Control Methods in IP Networks, In Advances in Multimedia, vol. 2013, Article ID 918930, 7 pages, ISSN: 1687-5680 (Print), ISSN: 1687-5699 (Online), doi:10.1155/2013/918930.

[3] B. E. A. Saleh, M. C. Teich. Fundamentals of photonics, A wiley-Interscience publication, pp. 739, TA1520.S2, 1991.

[4] J. Čuchran, R. Róka. Optocommunication systems and networks, STU Publishing house Bratislava, pp. 6-140, 2006.

[5] R. Róka, F. Čertík, Modeling of Environmental Influences at the signal transmission in the optical transmission medium, International Journal of Communication Networks and Information Security,vol. 4, No. 3. S146- 162. ISSN 2073-607X.

[6] R. Róka, F. Čertík. The Nonlinear FWM Effect and its Influence on Optical Signals Utilized Different Modulation
Techniques in the WDM Transmission Systems, OK $2012-$ 24th Conference, Praha (Czech), ISBN 978-80-86742-36-6, 25.-26. 10. 2012

[7] F. Čertík, R. Róka. Nonlinear SPM and XPM Effects and their Influence on Optical Signals Utilized Different Modulation Techniques in WDM Transmission Systems, OK 2014 - 26th Conference, Praha (Czech), 23.-24. 10. 2014.

[8] E. Iannone, F. Matera, A. Mecozzi, M. Settembre. Nonlinear Optical Communication Networks, Johnwiley and sons, pp. 20 50, TK5103.59.N66, 1998.

[9] K. P. Ho. Phase-Modulated Optical Communication Systems, 2005 Springer Science+Business Media, Inc, pp. 111 - 180, ISBN 0-387-24392-5, ISBN 978-0387-24392-4.

[10] R. Hill. A First Course in Coding Theory, Oxford: Clarendon Press.

[11] W. Trappe, L. C. Washington. Introduction to cryptography: with coding theory, 2nd ed. New Jersey: Pearson Prentice Hall.

[12] J. H. van Lint. Introduction to coding theory, 3rd ed. Berlin: Springer Verlag.

[13] M. Bossert. Channel Coding For Telecommunications, New York: John Wiley Sons.

[14] R. Róka. Fixed Transmission Media, Technology and Engineering Applications of Simulink, InTech, Rijeka (Croatia), ISBN 978-953-51-0635-7, May 2012. 
[15] Samsung Fiberoptics:

http://www.samsung.com/global/business/fiberoptics/opticalfiber/single-mode

[16] ITU-T Rec. 656 approved in 2010: http://www.itu.int/rec/TREC-G.656-201007-I/en

[17] F. G. Xiong. Digital Modulation Techniques, ArtechHouse, INC., pp. 23-234, ISBN 0-89006-970-0, 2000.

[18] J. M. Kahn, K. P. Ho. Spectral Efficiency Limits and Modulation/Detection Techniques for DWDM Systems, IEEE. J. on
Sel. Topics in QuantumElectron, 10, 259-272 (2004), 2005.

[19] P. Shukla, K. P. Kaur. Performance Analysis of EDFA for different Pumping Configurations at High Data Rate, International Journal of Engineering and Advanced Technology (IJEAT), vol. 2, ISSN: 2249 - 8958, , Issue-5, June 2013.

[20] K. Willox. Q Factor: The Wrong Answer for Service Providers and NEMs, ITU-T Temporary Document 25 (WP 1/4), 19 April 2002. 